\title{
EFEITO DE FUNGOS MICORRÍZICOS ARBUSCULARES, DA ADUBAÇÃO FOSFATADA E DA ESTERILIZAÇÃO DO SOLO NO CRESCIMENTO DE MUDAS DE MARACUJ AZEIRO AMARELO(1)
}

\author{
U. M. T. CAVALCANTE ${ }^{(2)}$, L. C. MAIA(3), C. M. C. COSTA $^{(4)}$, \\ A. T. CAVALCANTE ${ }^{(5)} \&$ V. F. SANTOS ${ }^{(5)}$
}

\begin{abstract}
RESUMO
O efeito da inoculação com fungos micorrízicos arbusculares (FMA), da adubação com fósforo e da esterilização do solo sobre o crescimento de mudas de maracujazeiro amarelo foi avaliado em experimento, realizado em casa de vegetação, inteiramente casualizado em fatorial de $2 \times 3 \times 4$, com três repetições, utilizando potes de $1,6 \mathrm{dm}^{3}$, sendo: dois tratamentos de solo (esterilizado - SE e não esterilizado - SNE ), três tratamentos de $P\left(4,11\right.$ e $30 \mathrm{mg} \mathrm{dm}^{-3}$ de $\left.P\right)$ e quatro tratamentos de inoculação (Gigaspora al bida, Scutel lospora heterogama, inóculo misto de G. al bida, G. margarita, Acaulospora longula e S. heterogama e o controle não inoculado). Mudas inoculadas e cultivadas em SE apresentaram aumento significativo na altura, diâmetro do caule, número de folhas e de gavinhas a partir de $\mathbf{5 0}$ dias da inoculação (d.a.i.), com exceção das inoculadas com S. heterogama, que mostraram respostas tardias (70 d.a.i.). Maiores índices de colonização radicular e densi dade de esporos na rizosfera foram registrados em SE. A esterilização do solo afetou a concentração de $P$ na parte aérea das mudas, apenas no tratamento-controle e inoculado com $\mathrm{S}$. heterogama. Em geral, a esterilização do solo, a adubação fosfatada e a inoculação com FMA favoreceram o crescimento das mudas de maracujazeiro, sendo registradas interações da inoculação mi corrízica, esterilização do solo e adubação fosfatada.
\end{abstract}

Termos de indexação: fungos endomicorrízicos, fósforo, Gigaspora albida, Gigaspora margarita, Scutel lospora heterogama, Acaulospora Iongula.

\footnotetext{
(1) Parte da Tese de Doutorado da primeira autora. Recebi do para publicação em outubro de 2000 e aprovado em agosto de 2002.

(2) Professora do Departamento de Biologia, Universidade Federal Rural de Pernambuco - UFRPE. Rua Dom Manuel de Medeiros s/n, Dois Irmãos, CEP 51.172-900 Recife (PE). Bolsista da CAPES. E-mail: umaaze @ig.com.br

(3) Professora do Departamento de Micologia, Universidade Federal Rural de Pernambuco - UFPE. Bolsista do CNPq. E-mail: leonorcmaia@hotmail.com

(4) Estudante da Pós-Graduação em Biologia de Fungos, UFPE. Bolsista da CAPES.

(5) Pesquisador do Instituto de Pesquisa Agropecuária de Pernambuco - IPA/PE. Av. Gal. San Martin 1371, CEP 50761-000 Recife (PE).
} 


\title{
SUMMARY: EFFECT OF ARBUSCULAR MYCORRHIZAL FUNGI, PHOSPHORUS SUPPLY, AND SOIL STERILIZATION ON GROWTH OF YELLOW PASSION FRUIT SEEDLINGS
}

\begin{abstract}
Theeffects of inoculation with arbuscular mycorrhizal fungi (AMF), of soil sterilization, and of phosphorus fertilization on growth of yel low passion fruit wereevaluated in greenhouse conditions. Theexperimental outlay was a compl etely randomized $2 \times 3 \times 4$ factorial design with three replicates per treatment. Theapplied treatments, using $1.6 \mathrm{dm}^{3}$ pots, were: two soil treatments (sterilized-SS and unsterilized soil-US), threP treatments (soil with 4, 11 and $30 \mathrm{mg} \mathrm{dm}^{-3}$ of $\mathrm{P}$ ) and four AMF treatments (inoculation with either Gigaspora albida or Scutell lospora heterogama, with mi xed inocul um of G. al bida, G. margarita, Acaul ospora Iongula and S. heterogama and an uninoculated control). I noculated seedlings cul tivated in SS presented significant increases of height, stem diameter, number of leaves and daspers after the $50^{\text {th }}$ day of inoculation (d.a.i.), except for S. heterogama, which promoted latehost responses ( $70^{\text {th }}$ d.a.i. .). Higher root col onization and spore density on the rhizosphere were registered in SS. Only in the control and S. heterogama treatments P shoot concentrations were negatively affected by soil sterilization. In general, soil sterilization, P supply, and AMF inoculation enhanced the growth of yel low passion fruit. Interactions of mycorrhizal inoculation with soil sterilization and with phosphorus were observed.
\end{abstract}

Index terms: endomycorrhizal fungi, phosphorus, Acaulospora longula, Gigaspora albida, Gigaspora margarita, Scutellospora heterogama.

\section{INTRODUÇÃO}

O maracujazeiroamarel o (Passi flora edulis Sims. f. flavi carpa Deg.) produz frutos bastante apreciados pel o sabor e que apresentam alto valor nutritivo. A produção de mudas da cultura éfeita basicamentea partir de sementes, sendo recomendada a adição de substrato comercial, fumigado, misturado com esterco de curral e superfosfato simples (Ruggiero et al., 1996). Fungos micorrízicos arbusculares (FMA) podem constituir outra alternativa para incrementar a produção de mudas, reduzindo a necessidade de aplicação desses insumos, sobretudo considerando a dependência do maracujazeiro à micorrização (Caval cante et al., 2001).

Expandindo a zona de absorção da raiz, pelo desenvolvimento de hifas que se ramificam, explorando e absorvendo maior quantidade de nutrientes minerais do solo, os FMAs podem acarretar maior crescimento das plantas, em menos tempo (Bago et al., 1998). Além da melhoria na nutrição, a inocul ação com FM A tem sido responsável pelo melhor estabelecimento de mudas no transplantio para o campo, conferindo também mai or tol erância a patógenos do solo (Smith \& Read, 1997). A associação com FMA pode contribuir, ainda, para aumento do teor de fósforo na planta (Chu, 1993; Camargo et al., 1990).

O benefício da inoculação com F MA na produção de mudas tem sido comprovado em várias fruteiras, tais como aceroleira (Costa et al., 2001), bananeira (J aizme-Veja et al., 1997), ameixeira e macieira
(Fortuna et al., 1996), citros (Souza et al., 1997), mamoeiro (Weber \& Amorim, 1994; Lins et al., 1999; Trindade et al., 2001) e maracujazeiro (Soares \& Martins, 2000).

Este trabalho objetivou identificar, em solo natural e esterilizado, a dose mais adequada de P e os isolados de FMA mais promissores para o estabel ecimento e sucesso da simbiose na promoção do crescimento de mudas de maracujazei ro amarelo.

\section{MATERIAL E MÉTODOS}

Foi usado Latossolo Amarelo distrófico argissólico, com as seguintes características: $4 \mathrm{mg} \mathrm{dm}^{3}$ de $\mathrm{P} ; 0,10,1,90,0,70$ e $0,12 \mathrm{cmol}_{\mathrm{c}} \mathrm{dm}^{3} \mathrm{de}$ $\mathrm{Al}, \mathrm{Ca}, \mathrm{Mg}$ eK, respectivamente; $\mathrm{pH} 5,7 ; 1,3,13,2 \mathrm{e}$ $22,8 \mathrm{~g} \mathrm{dm}^{-3}$ de N, C e MO, respectivamente. O solo foi desinfestado com brometo de metila $(98 \%$ de brometo de metila e $2 \%$ de cloropicrina), 20 dias antes da utilização, e a umidade mantida em torno de $60 \%$ do VTP (Volume Total de Poros). Consideraram-setrês doses (baixa, média ealta) de fósforo: 4 (solo natural), 11 e $30 \mathrm{mg} \mathrm{dm}^{-3}$ de $\mathrm{P}$ no solo, estas obtidas com a adição de superfosfato simples, incorporado ao solo antes do plantio. A escolha das doses baseou-se no recomendado para adubação desta cultura (Caval canti et al., 1998).

Os inóculos consistiram de uma suspensão de esporos, extraídos de cultura em solo pelo método de peneiramento úmido (Gerdemann \& Nicolson, 
1963) e centrifugação(J enkins, 1964), de Gigaspora albida Schenck \& Smith (UFPE 01); Gigaspora margarita Becker \& Hall, Acaulospora longula Spain $\&$ Schenck e Scutell ospora heterogama (Nicolson \& Gerd.) Walker \& Sanders (UFPE 02, 08 e 12, respectivamente), mantidos em potes decultura com grama baiana (Paspal um notatum Flügge).

As sementes de maracujá foram desinfestadas com hipoclorito de sódio, a $20 \%$ do produto comercial ( $2 \%$ do princípio ativo), e colocadas em solo fumigado. A inoculação foi efetuada em plântulas com apenas uma fol ha verdadeira. Cada vaso $(1,6 \mathrm{~kg})$, com uma planta, recebeu 100 esporos de G. al bida, de S. heterogama ou da mistura de FMA (25 esporos decada um dos quatro isol ados). Filtrado dos solos onde os inóculos foram produzidos foi adicionado em todos os tratamentos, para reestabelecimento da microbiota original, com exceção dos FMAs.

A altura das plantas, o diâmetro do caule a $3 \mathrm{~cm}$ do solo, o número de fol has e o de gavinhas foram avaliados a cada dez dias, após a inoculação. Além destes, foram determinadas, por ocasião da colheita, aos 70 dias, a concentração de $P$ na parte aérea, a col onização das raízes pel os F M As e a densidade de esporos na rizosfera. As raízes foram diafanizadas e coradas (Phillips \& Hayman, 1970) para avaliação da colonização micorrízica, sendo usados 100 fragmentos de $1 \mathrm{~cm} / a m o s t r a$ de raiz (Giovanetti \& Mosse, 1980). Os esporos de FMA foram extraídos do solo por peneiramento úmido (Gerdemann \& Nicolson, 1963) e centrifugações em água esacarose (J enkins, 1964).

O delineamento foi inteiramentecasualizado, com três repetições, em esquema fatorial $(2 \times 3 \times 4)$, correspondendo, respectivamente, à condição de esterilização do solo (esterilizado ou não), à quantidade de fósforo $\left(4,11\right.$ e $30 \mathrm{mg} \mathrm{dm}^{3}$ de $P$ no solo) e à inoculação com: (a) G. albida; (b) S. heterogama; (c) mistura de FMA (G. albida, G. margarita, A. Iongula e S. heterogama); (d) controle (não inoculado). Os dados foram submetidos à análise de variância, utilizando-se o programa SANEST (Zonta et al., 1984), e as médias foram comparadas pelo teste de Tukey a $5 \%$. Para regressão, foram relacionados doses de $P$, tratamentos de inoculação e épocas de col heita, como variáveis dependentes.

\section{RESULTADOS E DISCUSSÃO}

O crescimento do maracujazeiro amarel o foi, em geral, favorecido a partir dos 50 dias da inoculação dos fungos. Até esse período, não houve diferença no crescimento das plantas entre os tratamentos. I nicial mente, as mel hores respostas ocorreram com a inoculação de G. al bi da e, ou, com inóculo misto e, no final do experimento, também com S. heterogama (Quadro 1). Os resultados indicaram queeste fungo aparentemente requer mais tempo para colonizar as raízes do maracujazeiro, o que resultou no efeito tardio da resposta à inoculação. Outra possibilidade é que tenha havido germinação de S. heterogama sem que esta tenha sido imediatamente seguida pela col onização e estabel ecimento da simbiose. Algumas espécies estão adaptadas a situações em que a germinação não é seguida, de imediato, pela col onização; também deve ser considerado o fato de ser o micéli o externo importante para a produção de esporos (Smith \& Read, 1997) eo não-desenvolvimento deste compromete a esporulação, reduzindo a reinfecção por estas estruturas. Graça et al. (1991) relataram que $\mathrm{S}$. heterogama foi ineficiente, em maracujazeiro amarelo, quando inoculado isoladamente, porém se mostrou efetivo, quando aplicado juntamente com Azospirillum brasiliense.

A eficiência de G. albida em promover o crescimento do maracujazeiro (isoladamente ou compondo o inóculo misto) foi observada já aos 50 dias; esse fungo possivelmente coloniza mais rápido que $\mathrm{S}$. heterogama e, ou, apresenta maior compati bilidade com o hospedeiro. Bécard \& Piché (1990) também observaram rápida col onização com um isolado de G. margarita. Em estudos com Passiflora ligularis L., Rodrigues et al. (1995) observaram melhor resposta quando as plantas foram inoculadas com Acaul ospora Iongula do que com A. foveata Trappe \& J anos e Glomus occul tum Walker.

Nestetrabalho, A. Iongula foi um dos componentes do inóculo misto. Apesar da reconhecida falta de especificidade na simbiose mi corrízica arbuscular, a eficiência do FMA está sob controle genético e é afetada pela espécie da planta e do fungo, além de ser dependente das condições ambientais (Bagyaraj, 1991; Declerck et al., 1995).

Foram observadas interações significativas entre a inoculação do FMA e a esterilização do solo. O desdobramento evidenciou, aos 50 dias, plantas com maiores valores de altura, quando inoculadas com G. albida e com a mistura de FMA em solo esterilizado e, aos 60 e 70 dias, em todos os tratamentos inoculados (Quadro 1 ). Soares \& Martins (2000), trabalhando também com maracujazeiro amarelo, consideraram que a prática de fumigação do solo não é recomendável, por eliminar a microbiota, tornando necessária a inoculação do FMA e, ou, a aplicação de doses el evadas de $P$, uma vez que essa planta é dependente da micorrização (Caval cante et al., 2001).

Aos 50 dias, em solo esterilizado, as mudas inoculadas com G. albida apresentaram maior diâmetro do caule que as do tratamento-controle e daquele com S. heterogama (Quadro 1); no entanto, aos 70 dias, o benefício da micorrização em relação a essa característica foi observado em todos os 
Quadro 1. Efeito de F MA e da esterilização do solo sobre o crescimento do maracujazeiro amarelo, 50, 60 e 70 dias após a inoculação, independentemente dos níveis de fósforo

\begin{tabular}{|c|c|c|c|c|}
\hline \multirow{2}{*}{$\begin{array}{l}\text { Tratamento de } \\
\text { inoculação }\end{array}$} & \multicolumn{2}{|c|}{ Altura $(\mathrm{cm})$} & \multicolumn{2}{|c|}{ Diâmetro do caule (mm) } \\
\hline & SE & SNE & SE & SNE \\
\hline & \multicolumn{4}{|c|}{50 dias } \\
\hline $\begin{array}{l}\text { Controle } \\
\text { G. al bida } \\
\text { S. heterogama } \\
\text { I nóculo misto }\end{array}$ & $\begin{array}{l}24,93 \pm 6,93 \mathrm{bA} \\
43,03 \pm 6,38 \mathrm{aA} \\
26,61 \pm 4,82 \mathrm{bA} \\
40,31 \pm 5,95 \mathrm{aA}\end{array}$ & $\begin{array}{l}18,23 \pm 2,88 a A \\
20,53 \pm 4,34 a B \\
20,99 \pm 3,98 a A \\
23,57 \pm 3,95 a B\end{array}$ & $\begin{array}{l}3,16 \pm 0,34 \mathrm{cA} \\
4,22 \pm 0,16 \mathrm{aA} \\
3,53 \pm 0,17 \mathrm{bcA} \\
3,93 \pm 0,2 \mathrm{abA}\end{array}$ & $\begin{array}{l}3,34 \pm 0,33 \mathrm{aA} \\
3,55 \pm 0,25 \mathrm{aB} \\
3,50 \pm 0,33 \mathrm{aA} \\
3,63 \pm 0,34 \mathrm{aA}\end{array}$ \\
\hline & \multicolumn{4}{|c|}{60 dias } \\
\hline $\begin{array}{l}\text { Controle } \\
\text { G. al bida } \\
\text { S. heterogama } \\
\text { I nóculo misto }\end{array}$ & $\begin{array}{l}44,48 \pm 12,27 \mathrm{bA} \\
81,11 \pm 6,25 \mathrm{aA} \\
56,57 \pm 4,31 \mathrm{bA} \\
74,63 \pm 8,2 \mathrm{aA}\end{array}$ & $\begin{array}{l}23,97 \pm 4,38 \mathrm{aB} \\
24,74 \pm 5,58 \mathrm{aB} \\
29,66 \pm 6,82 \mathrm{aB} \\
32,37 \pm 5,96 \mathrm{aB}\end{array}$ & $\begin{array}{l}3,44 \pm 0,35 \mathrm{bA} \\
4,77 \pm 0,08 \mathrm{aA} \\
4,32 \pm 0,22 \mathrm{aA} \\
4,58 \pm 0,28 \mathrm{aA}\end{array}$ & $\begin{array}{l}3,65 \pm 0,41 \mathrm{aA} \\
3,94 \pm 0,34 \mathrm{aB} \\
3,84 \pm 0,39 \mathrm{bB} \\
4,04 \pm 0,39 \mathrm{abB}\end{array}$ \\
\hline I nóculo misto & \multicolumn{4}{|c|}{70 dias } \\
\hline $\begin{array}{l}\text { Controle } \\
\text { G. al bida } \\
\text { S. heterogama } \\
\text { I nóculo misto }\end{array}$ & $\begin{array}{l}61,51 \pm 15,03 \mathrm{bA} \\
98,84 \pm 1,35 \mathrm{aA} \\
88,67 \pm 4,47 \mathrm{aA} \\
96,45 \pm 2,17 \mathrm{aA}\end{array}$ & $\begin{array}{l}32,32 \pm 6,62 \mathrm{aB} \\
32,34 \pm 7,96 \mathrm{aB} \\
38,81 \pm 9,26 \mathrm{aB} \\
44,75 \pm 9,27 \mathrm{aB}\end{array}$ & $\begin{array}{l}3,73 \pm 0,41 \mathrm{bA} \\
5,01 \pm 0,16 \mathrm{aA} \\
4,74 \pm 0,18 \mathrm{aA} \\
4,77 \pm 0,16 \mathrm{aA}\end{array}$ & $\begin{array}{l}3,91 \pm 0,45 \mathrm{aA} \\
4,18 \pm 0,37 \mathrm{aB} \\
3,98 \pm 0,43 \mathrm{aB} \\
4,28 \pm 0,45 \mathrm{aB}\end{array}$ \\
\hline \multirow{2}{*}{ I nóculo misto } & \multicolumn{2}{|c|}{$N^{\circ}$ folha } & \multicolumn{2}{|c|}{$\mathbf{N}^{\circ}$ gavinha } \\
\hline & \multicolumn{4}{|c|}{50 dias } \\
\hline $\begin{array}{l}\text { Controle } \\
\text { G. al bida } \\
\text { S. heterogama } \\
\text { I nóculo misto }\end{array}$ & $\begin{array}{r}7,11 \pm 1,10 \mathrm{bA} \\
9,83 \pm 0,53 \mathrm{aA} \\
9,05 \pm 0,51 \mathrm{aA} \\
10,19 \pm 0,36 \mathrm{aA}\end{array}$ & $\begin{array}{l}7,42 \pm 0,68 \mathrm{aA} \\
8,25 \pm 0,57 \mathrm{aB} \\
8,24 \pm 0,60 \mathrm{aA} \\
8,41 \pm 0,76 \mathrm{aB}\end{array}$ & $\begin{array}{l}\text { (1) } \\
(1) \\
(1) \\
(1)\end{array}$ & $\begin{array}{l}\text { (1) } \\
\text { (1) } \\
\text { (1) } \\
\text { (1) }\end{array}$ \\
\hline I nóculo misto & \multicolumn{4}{|c|}{60 dias } \\
\hline $\begin{array}{l}\text { Controle } \\
\text { G. al bida } \\
\text { S. heterogama } \\
\text { I nóculo misto }\end{array}$ & $\begin{array}{r}8,55 \pm 1,35 \mathrm{bA} \\
12,37 \pm 0,64 \mathrm{aA} \\
11,73 \pm 0,49 \mathrm{aA} \\
12,04 \pm 0,63 \mathrm{aA}\end{array}$ & $\begin{array}{l}8,01 \pm 0,63 a A \\
8,60 \pm 0,52 a B \\
9,38 \pm 0,55 a B \\
8,85 \pm 0,79 a B\end{array}$ & $\begin{array}{l}1,72 \pm 0,73 \mathrm{bA} \\
4,08 \pm 0,57 \mathrm{aA} \\
3,23 \pm 0,47 \mathrm{aA} \\
4,07 \pm 0,57 \mathrm{aA}\end{array}$ & $\begin{array}{l}0,48 \pm 0,24 \mathrm{aB} \\
0,42 \pm 0,37 \mathrm{aB} \\
0,93 \pm 0,45 \mathrm{aB} \\
1,04 \pm 0,43 \mathrm{aB}\end{array}$ \\
\hline I nóculo misto & \multicolumn{4}{|c|}{70 dias } \\
\hline $\begin{array}{l}\text { Controle } \\
\text { G. al bida } \\
\text { S. heterogama } \\
\text { I nóculo misto }\end{array}$ & $\begin{array}{l}10,43 \pm 1,48 \mathrm{bA} \\
14,15 \pm 0,70 \mathrm{aA} \\
13,85 \pm 0,48 \mathrm{aA} \\
14,28 \pm 0,60 \mathrm{aA}\end{array}$ & $\begin{array}{r}8,91 \pm 0,62 \mathrm{aB} \\
9,46 \pm 0,68 \mathrm{aB} \\
10,34 \pm 0,72 \mathrm{aB} \\
9,73 \pm 0,85 \mathrm{aB}\end{array}$ & $\begin{array}{l}2,79 \pm 1,17 \mathrm{bA} \\
5,77 \pm 0,61 \mathrm{aA} \\
5,14 \pm 0,49 \mathrm{aA} \\
6,48 \pm 0,50 \mathrm{aA}\end{array}$ & $\begin{array}{l}0,82 \pm 0,44 a B \\
0,55 \pm 0,52 a B \\
1,38 \pm 0,60 a B \\
1,57 \pm 0,63 a B\end{array}$ \\
\hline
\end{tabular}

Médias seguidas da mesma letra minúscula, na coluna, e maiúscula, na linha, não diferem significativamente pelo teste de Tukey a $5 \%$; SE - solo esterilizado; SNE - solo não esterilizado.

(1) Sem produção de gavinhas.

tratamentos inoculados. Resultados semelhantes foram observados em citros (Cardoso \& Lambais, 1993; Souza et al., 1997) eem macieiras (Plenchette et al., 1981), porém também há registros indicando que o diâmetro do caule não aumentou por causa da micorrização (Antunes \& Cardoso, 1990; Silva \& Siqueira, 1991; Silveira et al., 2002). Os resultados obtidos neste trabalho poderão ser relevantes para a propagação vegetativa do maracujazeiro, sugerida por Ruggiero et al. (1996), tendo em vista que a inoculação micorrízica contribuiu para aumentar, consideravelmente, o diâmetro do caule, possibilitando a anteci pação da enxertia.
No solo esterilizado, as mudas inoculadas com FMA tiveram maior número de fol has e gavinhas que o controle, apresentando mais de nove fol has aos 50 dias. Ruggiero et al. (1996) consideram que mudas com oito fol has e altura em torno de $20 \mathrm{~cm}$ estão aptas para o transplantio. No entanto, o número de folhas parece nãoser indicativo de vigor, tendo em vista que as plantas-controle, não inoculadas, apresentaram de sete a dez folhas (Quadro 1). Estas eram pouco desenvolvidas e exibiam coloração verde-escura, típica, no maracujazeiro, da deficiência em fósforo (Ruggiero et al., 1996). 
No solo não esterilizado, não houve diferença entre os tratamentos, com relação ao número de fol has (Quadro 1). Possivel mente, a competição com os outros microrganismos impediu que os FMAs inoculados fossem efetivos. Entretanto, Rodrigues et al. (1995) verificaram, em Passiflora ligularis L., que a desinfestação do solo prejudicou o desenvolvimento das plantas e a eficiência na aquisição de fertilizante fosfatado.

As gavinhas surgiram nas plantas inoculadas aos 40 dias; todavia, só após 60 dias, perceberam-se interações entre tratamento do sol o e inoculação com FMA. Maior produção de gavinhas ocorreu nas plantas inoculadas e mantidas em sol o esterilizado, enquanto os tratamentos em solo não esterilizado não diferiram (Quadro 1). O surgimento de gavinhas no maracujazeiro indica o último momento para o plantio no campo (Ruggiero et al., 1996) e, nas plantas inoculadas, isso ocorreu aos 40 dias (dados não apresentados), diferentemente do que ocorreu em plantas não inoculadas.
Houve interação entre $\mathrm{P} \times \mathrm{FMA}$ com regressão linear ajustada aos 50 e 70 dias para altura das plantas $(\mathrm{P} \times$ controlee $\mathrm{P} \times \mathrm{S}$. heterogama), indicando que o crescimento das plantas não inoculadas, ou inoculadas com S. heterogama, foi dependente da fertilização fosfatada. Por outro lado, regressão hiperbólica foi ajustada entre $P \times G$. al bida e $P \times$ inóculo misto, indicando crescimento das mudas inoculadas com estes fungos, quando cultivadas em solo com até $30 \mathrm{mg} \mathrm{dm}^{-3}$ de P (Quadro 2).

Interações entre $P$ no solo e tratamentos de inoculação foram observadas com relação ao diâmetro do caule, aos 60 dias, com regressãolinear ajustada para $\mathrm{P} \times$ controle e regressão hiperbólica para $\mathrm{P} \times \mathrm{FMA}$ (Quadro 2). Também houveinteração entre $P \times F M A$ para o número de folhas, com regressão linear, a partir dos 50 dias, entre $P \times$ controle e, a partir dos 60 dias, entre $P \times$ S. heterogama. Regressão hiperbólica foi obtida entre $P \times G$. albida eP $\times$ inóculo misto (50 e 60 dias) (Quadro 2).

Quadro 2. E quação de regressão ajustada entre as doses de $P$ ( $\mathrm{mg} \mathrm{dm}^{-3} \mathrm{de} P$ no solo) e F MA, para variáveis de crescimento de mudas de maracujazeiro amarelo

\begin{tabular}{|c|c|c|c|}
\hline Variável de crescimento & Tratamento com F MA & E quação & $\mathbf{R}^{2}$ \\
\hline \multicolumn{4}{|c|}{50 dias } \\
\hline \multirow[t]{4}{*}{ Altura $(\mathrm{cm})$} & Controle & $\hat{Y}=4,67+1,13 x$ & 0,98 \\
\hline & S. heterogama & $\hat{Y}=9,87+0,93 x$ & 0,93 \\
\hline & G. albida & $\hat{Y}=49,03-140,64 / x$ & 0,98 \\
\hline & I nóculo misto & $\hat{Y}=49,08-137,35 / x$ & 0,99 \\
\hline \multirow[t]{4}{*}{ Número de folhas } & Controle & $\hat{\mathrm{Y}}=2,29+0,03 \mathrm{x}$ & 0,76 \\
\hline & S. heterogama & $\hat{\mathrm{Y}}=10,61-15,31 / \mathrm{x}$ & 0,99 \\
\hline & G. albida & $\hat{Y}=10,91-14,68 / x$ & 0,95 \\
\hline & I nóculo misto & $\hat{Y}=11,29-15,58 / x$ & 0,91 \\
\hline \multirow{5}{*}{ Diâmetro do caule (mm) } & $60 d$ & & \\
\hline & Controle & $\hat{\mathrm{Y}}=2,26+0,08 \mathrm{x}$ & 0,84 \\
\hline & S. heterogama & $\hat{Y}=5,12-8,31 / x$ & 0,99 \\
\hline & G. albida & $\hat{\mathrm{Y}}=5,08-5,81 / \mathrm{x}$ & 0,97 \\
\hline & Inóculo misto & $\hat{Y}=5,48-9,32 / x$ & 0,99 \\
\hline \multirow[t]{4}{*}{ Número de folhas } & Controle & $\hat{Y}=2,47+0,03 x$ & 0,74 \\
\hline & S. heterogama & $\hat{Y}=3,13+0,01 x$ & 0,75 \\
\hline & G. albida & $\hat{\mathrm{Y}}=12,41-15,66 / \mathrm{X}$ & 0,98 \\
\hline & I nóculo misto & $\hat{\mathrm{Y}}=12,79-18,67 / \mathrm{x}$ & 0,95 \\
\hline \multirow{5}{*}{ Altura $(\mathrm{cm})$} & $80 \mathrm{~d}$ & & \\
\hline & Controle & $\hat{\mathrm{Y}}=16,75+2,01 \mathrm{X}$ & 0,74 \\
\hline & S. heterogama & $\hat{\mathrm{Y}}=47,78+1,06 \mathrm{x}$ & 0,99 \\
\hline & G. albida & $\hat{Y}=76,37-86,44 / x$ & 0,78 \\
\hline & Inóculo misto & $\hat{Y}=87,44-134,96 / x$ & 0,88 \\
\hline \multirow[t]{2}{*}{ Colonização das raízes (\%) } & G. albida & $\hat{Y}=61,12+0,88 x$ & 0,99 \\
\hline & Inóculo misto & $\hat{Y}=62,26+1,13 x$ & 0,90 \\
\hline $\begin{array}{l}\text { Densidade de esporos } \\
\text { (nø/100 g solo) }\end{array}$ & I nóculo misto & $\hat{Y}=93,48+6,28 x$ & 0,98 \\
\hline
\end{tabular}


Na colonização das raízes, foi possível ajustar uma regressão linear entre $P \times G$. al bi da e $P \times$ inóculo misto, enquanto na densidade de inócul o esse ajuste foi entre $\mathrm{P} \times$ inóculo misto (Quadro 2 ), indicando a importância desse nutriente na relação simbiótica e na multiplicação do fungo.

Aos 70 dias, apenas as mudas cultivadas em solo esterilizado e que receberam inóculo misto apresentaram maior concentração de $\mathrm{P}$ que o controle. No solo não esterilizado, as plantas inoculadas com a mistura deFMA apresentaram menor concentração de $P$ na parte aérea que as inoculadas com S. heterogama ou não inoculadas. A concentração de $P$ foi maior nas plantas cultivadas em solo não esterilizado do que no esterilizado apenas nos tratamentos-controle (com fungos nativos) e com S. heterogama (Quadro 3). Cuenca et al. (1990) observaram que FMAs nativos ocasionaram aumento na absorção de $\mathrm{P}$ e de outros nutrientes, em plântulas de cacau.

Os valores de $P$ na parte aérea das plantas estavam abaixo daquele considerado adequado $(0,5 \%)$ para o maracujazeiro amarelo (Ruggiero et al., 1996). A absorção de $\mathrm{P}$ pode ter maior impacto no início da colonização, quando as hifas externas estão em densidade mais baixa (O'K eefe \& Sylvia, 1992). Por outro lado, Karagiannidis et al. (1997) observaram, em videiras, que o teor de $\mathrm{P}$ nas fol has não estava diretamente relacionado com o grau de col onização mi corrízica das raízes nem como teor de $\mathrm{P}$ no solo, embora as folhas tenham apresentado ótimo suprimento de P. Em citros, não se observou relação entre teores de $\mathrm{P}$ nas fol has e no solo, nas plantas micorrizadas, enquanto, no controle, o aumento de $\mathrm{P}$ no solo resultou em maior teor de $\mathrm{P}$ foliar (Davis \& Fucik, 1986).

Soares \& Martins (2000) obtiveram aumentos significativos na produção de matéria seca e no teor de alguns nutrientes, incluindo $P$, em mudas de maracujazeiro amarelo inoculadas com Glomus clarum Nicolson \& Schenck, G. fasciculatum (Thaxter) Gerd. \& Trappe emend. Walker \& Koske ecom F MA nativos. Esses autores aplicaram os fungos associados a compostos fenólicos, mas verificaram que estes não eram indispensáveis para estimular e acelerar a colonização radicular promovida por eles.

As espécies de FMA não contribuíram igual mente para aumentar a absorção de nutrientes e o crescimento da planta (Abbott \& Gazey, 1994). Silveira et al. (2002) não observaram alteração nos teores de $\mathrm{P}$ em porta-enxertos de abacateiro inoculados com seis espécies de FMA; entretanto, diferenças ocorreram na absorção de N, K, Ca, Mg e $\mathrm{Fe}$, conforme o FMA inoculado. É possível que o tempo de duração do experimento (70 dias) não tenha sido suficiente para detectar a maior concentração deP na parteaérea dos maracujazeiros inoculados. Em citros, só foram observadas diferenças no acúmulo de nutrientes 180 dias após o transplantio (Cardoso \& Lambais, 1993). Outra alternativa, para explicar a ausência de diferenças na concentração de $P$ na parteaérea entreas mudas inoculadas e não inoculadas, seria que as doses de $P$ aplicadas não foram suficientes para que o nutriente fosse absorvido etransportado em maior quantidade para a parte aérea do maracujazeiro.

A esterilização do solo favoreceu a colonização das raízes ea densidade de esporos na rizosfera das plantasinoculadas (Quadro 3). Resultados semel hantes foram obtidos por Camargo et al. (1990) em portaenxertos de citros. A ausência de organismos prejudiciais (Kleinschmidt \& Gerdemann, 1972) e, ou, de competidores, no solo esterilizado contribuiu, possivel mente, para essas respostas.

Nos solos com ou sem esterilização, todos os tratamentos inoculados apresentaram maior percentagem de col onização que o controle. Por outro lado, mesmo na presença de FMA nativo, no solo não esterilizado, os fungos inoculados promoveram maior col onização (Quadro 3), sendotambém mais infectivos. Antunes \& Cardoso (1990) não observaram diferenças significativas na colonização radicular entre porta-enxertos de citros inoculados e não inoculados, quando em sol o não esterilizado.

Quadro 3. Efeito de FMA e da esterilização do solo sobre a concentração de P, colonização de raízes e densidade de esporos na rizosfera do maracujazeiro amarelo, 70 dias após a inoculação

\begin{tabular}{|c|c|c|c|c|c|c|}
\hline \multirow{2}{*}{$\begin{array}{l}\text { Tratamento de } \\
\text { inoculação }\end{array}$} & \multicolumn{2}{|c|}{ Concentração de $P$ na parte aérea } & \multicolumn{2}{|c|}{ Colonização da raiz } & \multicolumn{2}{|c|}{ Densidade de esporo } \\
\hline & SE & SNE & SE & SNE & SE & SNE \\
\hline & & \multicolumn{2}{|c|}{ no/100 g de solo } \\
\hline Não inoculado & $0,051 \pm 0,004 \mathrm{bB}$ & $0,096 \pm 0,010 \mathrm{aA}$ & $1,77 \pm 1,12 \mathrm{cB}$ & $29,03 \pm 7,70 \mathrm{bA}$ & $5,11 \pm 3,21 b B$ & $57,33 \pm 25,02$ aA \\
\hline G. albida & $0,070 \pm 0,004 \mathrm{abA}$ & $0,085 \pm 0,010 \mathrm{abA}$ & $92,22 \pm 2,09 \mathrm{abA}$ & $56,51 \pm 8,46 \mathrm{aB}$ & $418,88 \pm 56,96 \mathrm{aA}$ & $72,88 \pm 20,75 a B$ \\
\hline S. heterogama & $0,066 \pm 0,007 \mathrm{abB}$ & $0,090 \pm 0,000 \mathrm{aA}$ & $80,66 \pm 5,00 \mathrm{bA}$ & $49,11 \pm 7,86 \mathrm{aB}$ & $477,77 \pm 68,48 \mathrm{aA}$ & $44,66 \pm 10,45 \mathrm{aB}$ \\
\hline I nóculo misto & $0,075 \pm 0,004 \mathrm{aA}$ & $0,064 \pm 0,008 \mathrm{bA}$ & $95,33 \pm 1,66$ aA & $63,11 \pm 10,10 \mathrm{aB}$ & $294,66 \pm 51,71 \mathrm{aA}$ & $80,88 \pm 26,73 a \mathrm{aB}$ \\
\hline
\end{tabular}

Médias seguidas da mesma letra minúscula, na coluna, e maiúscula, na linha, não diferem significativamente pelo teste de Tukey a $5 \%$. SE - solo esterilizado; SNE - solo não esterilizado. 
No solo esterilizado, a densidade de esporos na rizosfera das plantas inoculadas foi maior do que no controle, enquanto, no solo não esterilizado, não houve diferença entre os tratamentos, possivelmente porque os fungos nativos foram tão eficientes na produção de esporos quanto os introduzidos (Quadro 3).

A esterilização do solo não impediu que, aos 70 dias, fossem observadas estruturas de FMA nas raízes e no solo da rizosfera das plantas-controle (Quadro 3). I sso pode ter sido conseqüência da col onização de propágulos infectivos que escaparam à esterilização, de algum mecanismo de dispersão, levando esporos para o solo esterilizado, ou da presença dealguns propágulos introduzidos por meio da sol ução do sol o onde foram produzidos os inóculos, que foi colocada nas plantas-controle.

Entretanto, esta última possibilidade é pouco provável, tendo em vista a ausência de esporos de FMA na solução do solo, conforme constatado. Reinvasão deFMA em solo esterilizado com brometo de metila pode ocorrer após seis ou mais meses em presença de um hospedeiro susceptível (Ridings et al., 1977). Menge (1982) não detectou propágulos de FMA uma semana após a fumigação de sol o com brometo de metila, porém baixas populações de propágulos infectivos foram detectadas após dois a 13 meses. O autor concluiu que raramente ocorre destruição completa dos F MA com esse tratamento. Aumento da população de FMA nativo em solo esterilizado com brometo de metila pode ocorrer em decorrência do crescimento ativo do hospedei ro e dos propágulos fúngi cos que escaparam à esterilização ou que foram introduzidos durante o experimento por mecanismos de dispersão (Walker et al., 1982).

\section{CONCLUSÕES}

1. A esterilização do sol o e a adubação fosfatada, até $30 \mathrm{mg} \mathrm{dm}^{-3}$, beneficiam as respostas de crescimento do maracujazeiro à inoculação com FMA, assim como a colonização de raízes e a produção de esporos na rizosfera.

2. Em geral, o benefício da simbiose micorrízica para o crescimento do maracujazeiro começa a ser evidenciado 50 dias após a inoculação, dependendo do isolado deFMA; S. heterogama promoveresposta tardia (70 dias), em comparação com G. al bida ecom o inóculo misto utilizado.

\section{LITE RATURA CITADA}

ABBOTT, L.K. \& GAZEY, C. An ecological view of the formation of VA mycorrhizas. Plant Soil, 159:69-78, 1994.

ANTUNES, V. \& CARDOSO, E.J.N. O fósforo e a micorriza vesículo arbuscular no crescimento de porta-enxertos de citros cultivados em solo natural. R. Bras. Ci. Solo, 14:277282, 1990.
BAGO, B.; AZCÓN-AGUILAR, A.; GOULET, A. \& PICHÉ, Y. Branched absorbing structures (BAS): a feature of the extraradical mycelium of symbiotic arbuscular mycorrhizal fungi. New Phytol., 139:375-388, 1998.

BAGYARAJ , D.J . Use of VA mycorrhiza in practical agriculture. In: REUNIÃO BRASILEIRA SOBRE MICORRIZAS, 5., Mendes, 1991. Resumos. I taguaí, EMBRAPA-Centro Nacional de Pesquisa em Biologia do Solo, 1991. p.81-92.

BÉCARD, G. \& PICHÉ, Y. Physiological factors determining vesicular-arbuscular formation in host and nonhost $\mathrm{Ri} \mathrm{T}$ DNA transformed roots. Can. J. Bot., 68:1260-1264, 1990.

CAMARGO, I.P.; SOUZA, M.; CARVALHO,J .G. \& OLIVEIRA, E. Doses e fontes de fósforo e de fungos micorrízicos sobre a nutrição mineral do limoeiro 'Cravo' até a repicagem. Pesq. Agropec. Bras., 25:1465-1470, 1990.

CARDOSO, E.J.B.N. \& LAMBAIS, M.R. Efeito de aldicarb e fosetil-al no desenvolvimento e na colonização micorrízica detangerina 'Cleópatra'. R. Bras. Ci. Solo, 17:179-184, 1993.

CAVALCANTI, F.J .A.; SANTOS, J.C.P.; PEREIRA, J.R.; LEITE, J .P.;SILVA, M.C.L.;FREIRE, F.J .;SILVA, D.J .;SOUSA, A.R.; MESSIAS, A.S.; FARIA, C.M.B.; BURGOS, N.; LIMA J ÚNIOR, M.A; GOMES, R.V.; CAVALCANTI, A.C. \& LIMA, J.F.W.F. Recomendações de adubação para o Estado de Pernambuco (2ª aproximação). 2.ed. Recife, IPA, 1998. 198p.

CAVALCANTE, U.M.T.; MAIA, L.C.; COSTA, C.M.C. \& SANTOS, V.F. Mycorrhizal dependency of passion fruit (Passiflora edulis f. flavicarpa). Fruit, 56:317-324, 2001.

CHU, E.Y. Inoculação de fungos micorrízicos em plântulas de acerola (Malpighia glabra L.) Belém, EMBRAPA/CPATU, 1993. 15p. (Boletim de Pesquisa, 149)

COSTA, C.M.C.; MAIA, L.C.; CAVALCANTE, U.M.T. \& NOGUEIRA, R.J.M.C. Influência de fungos micorrízicos arbusculares sobre o crescimento de dois genótipos de aceroleira (Malpighia emarginata D.C.). Pesq. Agropec. Bras., 36:893-901, 2001.

CUENCA, G.; HERRERA, R. \& MENESES, E. Effects of VA mycorrhiza on the growth of cacao seedlings under nursery conditions in Venezuela. Plant Soil, 126:71-78, 1990.

DAVIS, R.M. \& FUCIK, J.E. Effect of girdling sour orange seedlings on mycorrhizal development. HortScience, 21:302-304, 1986.

DECLERCK，S.; PLENCHETTE，C.\& STRULLU, D.G. Mycorrhizal dependency of banana (Musa acuminata, grupo AAA) cultivar. Plant Soil, 176:183-187, 1995.

FORTUNA, P.; CITERNESI, A.S.; MORINI, S.; VITAGLIANO, C. \& GIOVANETTI, M. Influence of arbuscular mycorrhizae and phosphate fertilization on shoot apical growth of micropropagated apple and plum rootstocks. Tree Physiol., 16:757-763, 1996.

GERDEMANN, J.W. \& NICOLSON, T.H. Spores of mycorrhizal Endogone species extracted from soil by wet sieving and decanting. Trans. Br. Mycol. Soc.,46:235-244, 1963.

GIOVANETTI, M.\& MOSSE, B. An evaluation of techniques for measuring vesicular arbuscular mycorrhizal infection in roots. New Phytol., 84:489-500, 1980. 
GRAÇA, J .P.; MACHADO, J.P.O.; RUGGIERO, C.\& ANDRIOLO, J.L. Eficiência de fungos endomicorrízicos e da bactéria Azospi rillum brasiliensesobre o desenvol vimento de mudas de maracujá (Passiflora edulisf. flavicarpa). R. Bras. Frutic., 13:125-130, 1991.

J AIZME-VEGA, M.C.;TENOURY, P.; PINOCHET, J \& \& AUMOT, $M$. Interactions between the root knot nematode Meloidogyne incognita and Glomus mosseae in banana. Plant Soil, 196:27-35, 1997.

JENKINS, W.R. A rapid centrifugal-floatation technique for separating nematodes from soil. Plant Dis. Rep., 48:692, 1964.

KARAGIANNIDIS, N.; VELEMIS, D. \& STAVROPOULOS, N. Root colonization and spore population by VA-mycorrhizal fungi in four grapevine rootstocks. Vitis, 36:57-60, 1997.

KLEINSCHMIDT, G.D. \& GERDEMANN, J.W. Stunting of citrus seedlings in fumigated nursery soils related to the absence of endomycorrhizae. Phytopathology, 62:1447-1453, 1972.

LINS, C.E.L.; AGUIAR, R.L.F.; CAVALCANTE, U.M.T. \& MAIA, L.C. Influência da adubação com esterco bovino e inoculação de fungos micorrízicos arbusculares no crescimento de mudas de Carica papaya L. (var. Formosa). Acta Bot. Bras., 13:257-261, 1999.

MENGE, J.A. Effect of soil fumigant and fungicides on vesiculararbuscular fungi. Phytopathology, 72:1125-1182, 1982.

O'KEEFE, D.M. \& SYLVIA, D.M. Chronology and mechanisms of $\mathrm{P}$ uptake by mycorrhizal sweet potato plants. New Phytol., 122:651-659, 1992.

PHILLIPS, J.M. \& HAYMAN, D.S. I mproved procedures to clearing roots and staining parasitic and vesiculararbuscular mycorrhizal fungi for rapid assessment of infection. Trans. Br. Mycol. Soc., 55:158-160, 1970.

PLENCHETTE, C.; FURLAN, V. \& FORTIN, J.A. Growth stimulation of apple in unsterilized soil under field conditions with VA mycorrhiza inoculation. Can. J. Bot., 59:2003-2008, 1981.

RIDINGS, W.H.; SCHENCK, N.C.; SNELL, R.R.; KEEN, W.M. \& CORNELL, J.A. Reinvasion of methyl bromidetreated soil by soil-born fungi and their subsequent effect on citrus seedlings growth. Proc. Florida State Hortic. Soc., 90:7074, 1977.
RODRIGUES, G.A.M.; HURTADO, M. \& PRAGER, M.S. Inoculacion degranadilla Passiflora ligularis L. Acta Agron., 45:89-98, 1995.

RUGGIERO, C.; SÃO J OSÉ, A.R.;VOLPE, C.A.; OLIVEIRA,J .C.; DURIGAN, J.F.; BAUMGARTNER, J.G.; SILVA, J.R.; NAKAMURA, K.; FERREIRA, M.E.; KAVATI, R. \& PEREIRA, V.P. Maracujá para exportação: aspectos técnicos da produção. Ministério da Agricultura e doAbastecimento, Secretaria de Desenvolvimento Rural. Programa de Apoio à Produção e Exportação de Frutas, Hortaliças, Flores e Plantas Ornamentais. Brasília: E MBRAPA-SPI, 1996. 64p. (Publicações Técnicas FRUPEX, 19)

SILVA, L.F.C. \& SIQUEIRA, J.O. Crescimento e teores de nutrientes de mudas de abacateiro, mangueira e mamoei ro sob influência de diferentes espécies de fungos micorrízicos vesículo-arbusculares. R. Bras. Ci. Solo, 15:283-285, 1991.

SILVEIRA, S.V.; SOUZA, P.V.D. \& KOLLER, O.C. Influência de fungos micorrízicos arbusculares sobre o desenvolvimento vegetativo de porta-enxertos de abacateiro. Pesq. Agropec. Bras., 37:303-309, 2002.

SMITH, S.E. \& READ, D.J . Mycorrhizal symbiosis. London, Academic Press, 1997. 605p.

SOARES, A.C.F. \& MARTINS, M.A. Influência de fungos micorrízicos arbusculares, associada à adição de compostos fenólicos, no crescimento de mudas de maracujazeiro amarelo (Passiflora edulis f. flavicarpa). R. Bras. Ci. Solo 24:731-740, 2000.

SOUZA, P.V.D.; BERJ ON, M.A.; ORENGA, V.A. \& FONFRIA, $M$.A. Desenvolvimento do citrange Troyer' infectado com fungo micorrízico, em dois substratos de cultivo. Pesq. Agropec. Bras., 32:1039-1045, 1997.

TRINDADE, A.V.; SIQUEIRA, J.O. \& ALMEIDA, E.F. Dependência micorrízica de variedades comerciais de mamoeiro. Pesq. Agropec. Bras., 36:1485-1494, 2001.

ZONTA, E.P.; MACHADO,A.A.\& SILVEIRA J ÚNIOR, P. Sistema de análise estatística para microcomputadores (SANEST). Pel otas, Departamento de Matemática e Estatística, 1984. 151p.

WALKER, C.; MIZE, C.W. \& MCNABB J R., H.S. Populations of endogonaceus fungi at two locations in central Iowa. Can. J. Bot., 60:2518-2529, 1982.

WEBER, O.B. \& AMORIM, S.M.C. Adubação fosfática e inoculação de fungos micorrízicos vesicular arbusculares em mamoeiro 'Solo'. R. Bras. Ci. Solo, 18:187-191, 1994. 